biofilm, and comparison with various heparin solutions. J Antimicrob Chemother 2009;63:937-945.

\section{Gastrointestinal Selective Capacity of Doripenem, Meropenem, and Imipenem for Carbapenem-Resistant Gram-Negative Bacilli in Treated Patients with Pneumonia}

To the Editor-Multidrug-resistant gram-negative bacilli (GNB) have emerged as major infectious threats and therapeutic challenges for physicians worldwide. ${ }^{1,2}$ Infections with these multidrug-resistant pathogens have been associated with poor patient outcomes. ${ }^{3,4}$ Data on the emergence of carbapenem-resistant (CR) GNB in gastrointestinal flora and the selective capacity of carbapenem exposure are limited. We conducted a feasibility trial to evaluate the gastrointestinal selective capacity of 3 carbapenems for CR Acinetobacter baumannii, CR Pseudomonas aeruginosa, and CR Stenotrophomonas maltophilia among patients treated for healthcareassociated pneumonia. These findings on the emergence of multidrug-resistant GNB contribute to the current understanding of the selective capacity of gastrointestinal flora after carbapenem exposure.

From October 31, 2009, through August 31, 2010, all patients who were admitted to the medical intensive care unit (ICU) at Thammasat University Hospital with healthcare- associated pneumonia were approached for study participation. Consecutive consenting adults were enrolled. By means of a computer-generated list, patients were randomly assigned at a $1: 1: 1$ ratio to receive imipenem, meropenem, or doripenem at admission after enrollment. Clinical criteria for healthcare-associated pneumonia were the same as described elsewhere. ${ }^{5}$ Rectal swab specimens for culture were obtained at admission, on day 14 , and on day 28 . Patients who tested positive for enteric CR-GNB at admission or patients who died before day 14 were excluded. Prestudy baseline ICU rates of CR A. baumannii, CR P. aeruginosa, and CR S. maltophilia colonization or infection were $0.85,0.14$, and 0.05 cases per 1,000 patient-days, respectively. Rectal swab specimens were transported and processed within 1 hour of procurement for culture on MacConkey agar plates. Bacterial colonies suspected of being $A$. baumannii, $P$. aeruginosa, or S. maltophilia were identified using standard microbiological techniques. The minimum inhibitory concentrations (MICs) of all representative isolates were determined for the 3 study drugs by E-test ( $\mathrm{AB}$ bioMerieux), in accordance with the manufacturer's protocol. Susceptibility results were interpreted according to Clinical and Laboratory Standards Institute breakpoints. ${ }^{6}$ Laboratory personnel were masked to treatment assignments.

During the study period, 69 patients were screened for study participation, and 60 met the study criteria for participation and follow-up (20 patients per drug group). Excluded patients included 4 who tested positive for enteric CR-GNB at admission ( 2 positive for CR $A$. baumannii and 2 positive

TABLE 1. Characteristics of 60 Study Subjects with Healthcare-Associated Pneumonia and the Emergence of Carbapenem-Resistant (CR) Enteric Flora after Exposure to Carbapenems

\begin{tabular}{lccc}
\hline Variable & $\begin{array}{c}\text { Imipenem } \\
(n=20)\end{array}$ & $\begin{array}{c}\text { Meropenem } \\
(n=20)\end{array}$ & $\begin{array}{c}\text { Doripenem } \\
(n=20)\end{array}$ \\
\hline Characteristics & & & \\
$\quad$ Age, years & $51(31-65)$ & $50(25-69)$ & $49(28-67)$ \\
Male sex & $12(60)$ & $13(65)$ & $13(65)$ \\
Comorbid conditions & & & \\
$\quad$ Diabetes & $6(30)$ & $5(25)$ & $6(30)$ \\
$\quad$ COPD & $5(25)$ & $4(20)$ & $5(25)$ \\
$\quad$ Chronic liver disease & $4(20)$ & $4(20)$ & $3(15)$ \\
$\quad$ Chronic kidney disease & $5(25)$ & $5(25)$ & $4(20)$ \\
$\quad$ Neurological disease & $3(15)$ & $3(15)$ & $2(10)$ \\
APACHE II score, median & 17 & 16 & 19 \\
Duration of study therapy, days & $7(6-16)$ & $6(5-16)$ & $7(6-15)$ \\
Outcomes & & & \\
Day 14 after treatment & $4(20)$ & $4(20)$ & $3(15)$ \\
$\quad$ CR Acinetobacter baumannii & $6(30)$ & $6(30)$ & $0(0)^{\mathrm{a}}$ \\
$\quad$ CR Pseudomonas aeruginosa & $1(5)$ & $1(5)$ & $1(5)$ \\
$\quad$ CR Stenotrophomonas maltophilia & & & \\
Day 28 after treatment & $4(20)$ & $4(20)$ & $3(15)$ \\
$\quad$ CR A. baumannii & $5(25)$ & $5(25)$ & $0(0)^{\mathrm{a}}$ \\
CR P. aeruginosa & $1(5)$ & $1(5)$ & $1(5)$ \\
CR S. maltophilia & & & \\
\hline & &
\end{tabular}


for CR $P$. aeruginosa) and 5 who died before day 14 . The median age of patients was 51 years (range, 31-69 years), the median duration of carbapenem therapy was 7 days (range, 5-16 days), and no enrolled patients had enteric CR-GNB infection or colonization at admission. Patient characteristics are summarized in Table 1 . On day 14, CR A. baumannii, $\mathrm{CR} P$. aeruginosa, and CR $S$. maltophilia were detected in 4 subjects $(20 \%), 6$ subjects $(30 \%)$, and 1 subject $(5 \%)$, respectively, in the imipenem group, compared with 4 subjects $(20 \%), 6$ subjects $(30 \%)$, and 1 subject $(5 \%)$, respectively, in the meropenem group and 3 subjects ( $15 \%), 0$ subjects $(0 \%)$, and 1 subject (5\%), respectively, in the doripenem group. On day 28, CR A. baumannii, CR $P$. aeruginosa, and CR $S$. maltophilia were detected in 4 subjects $(20 \%), 5$ subjects $(25 \%)$, and 1 subject $(5 \%)$, respectively, in the imipenem group, compared with 4 subjects (20\%), 5 subjects (25\%), and 1 subject $(5 \%)$ in the meropenem group and 3 subjects $(15 \%), 0$ subjects $(0 \%)$, and 1 subject $(5 \%)$ in the doripenem group.

Overall, there were no differences in the gastrointestinal selective capacity of the 3 carbapenems for the emergence and detection of CR A. baumannii or CR S. maltophilia 14 and 28 days after treatment (Table 1). However, on day 14, selection for CR $P$. aeruginosa was found less frequently in the doripenem group than in the imipenem group $(0 \%$ vs $30 \% ; P=.01)$ and the meropenem group ( $0 \%$ vs $30 \%$; $P=.01$ ). On day 28 , selection for CR $P$. aeruginosa was found less frequently in the doripenem group than in the imipenem group ( $0 \%$ vs $25 \% ; P=.04)$ and the meropenem group $(0 \%$ vs $25 \% ; P=.04$ ) (Table 1 ). The $\mathrm{MIC}_{90}$ values for carbapenems were $>32 \mathrm{mg} / \mathrm{L}$ for both CR A. baumannii and CR S. maltophilia isolates in all 3 groups. They were 16 and 8 $\mathrm{mg} / \mathrm{L} 14$ days after treatment and 16 and $8 \mathrm{mg} / \mathrm{L} 28$ days after treatment for $\mathrm{CR} P$. aeruginosa in the imipenem and meropenem groups, respectively; MIC data were not available for the doripenem group on days 14 and 28.

Although limited by a small sample size and the selected patient population, our study findings suggest that there are no differences in the emergence of CR A. baumannii or CR S. maltophilia after exposure to imipenem, meropenem, or doripenem for treatment of healthcare-associated pneumonia. However, the emergence of CR $P$. aeruginosa was less frequent among subjects who received doripenem. Additional studies will be important to promote understanding of the gastrointestinal selective capacity of carbapenems and other anti-infective agents for the emergence of multidrug-resistant gram-negative pathogens and their contributory role in the transmission dynamics of drug resistance.

\section{ACKNOWLEDGMENTS}

Financial support. This investigation was supported in part by the Jensen Company, Merck, and AstraZeneca. The Jensen Company provided doripenem, disk susceptibility tests, and E-tests for doripenem; Merck provided disk susceptibility tests and E-tests for imipenem; and AstraZeneca provided disk susceptibility tests and E-tests for meropenem. This study was also supported by the National Research University Project of the Thailand Office of Higher Education Commission (to A.A.).

Potential conflicts of interest. All authors report no conflicts of interest relevant to this article.

\section{Anucha Apisarnthanarak, $\mathrm{MD}^{1}$ Pattarachai Kiratisin, $\mathrm{MD}, \mathrm{PhD}^{2}$ Piyaporn Apisarnthanarak, $\mathrm{MD}^{3}$ Linda M. Mundy, MD, $\mathbf{P h D}^{4}$}

Affiliations: 1. Division of Infectious Diseases, Thammasat University Hospital, Pratumthani, Thailand; 2. Department of Microbiology, Siriraj Hospital, Mahidol University, Bangkok, Thailand; 3. Department of Radiology, Siriraj Hospital, Mahidol University, Bangkok, Thailand; 4. LM Mundy, LLC, Bryn Mawr, Pennsylvania.

Address correspondence to Anucha Apisarnthanarak, MD, Division of Infectious Diseases, Thammasat University Hospital, Pratumthani, Thailand 12120 (anapisarn@yaboo.com).

Infect Control Hosp Epidemiol 2011;32(4):410-411

(C) 2011 by The Society for Healthcare Epidemiology of America. All rights reserved. 0899-823X/2011/3204-0021\$15.00. DOI: 10.1086/659252

\section{REFERENCES}

1. Peleg AY, Seifert H, Paterson DL. Acinetobacter baumannii: emergence of a successful pathogen. Clin Microbiol Rev 2008;21:538582.

2. Paterson DL. The epidemiological profile of infections with multidrug-resistant Pseudomonas aeruginosa and Acinetobacter species. Clin Infect Dis 2006;43(suppl 2):S43-S48.

3. Fishbain J, Peleg AY. Treatment of Acinetobacter infections. Clin Infect Dis 2010;51:79-84.

4. Kang CI, Kim SH, Kim HB, et al. Pseudomonas aeruginosa bacteremia: risk factors for mortality and influence of delayed receipt of effective antimicrobial therapy on clinical outcome. Clin Infect Dis 2003;37:745-751.

5. Micek ST, Kollef KE, Reichley RM, Roubinian N, Kollef ME. Health care-associated pneumonia and community acquired pneumonia: a single-center experience. Antimicrob Agents Chemother 2007;51:3569-3573.

6. Clinical and Laboratory Standards Institute (CLSI). Performance standards for antimicrobial susceptibility testing: nineteenth informational supplement. Document M100-\$19. Wayne, PA: CLSI, 2009.

\section{Intervention to Reduce the Incidence of Healthcare-Associated Methicillin-Resistant Staphylococcus aureus Infection in a Tertiary Care Hospital in Saudi Arabia}

To the Editor-The greatest success in controlling methicillinresistant Staphylococcus aureus (MRSA) has been in places that adhere to rigorous transmission-based control policies that include active surveillance culture testing to identify colonized patients and strict application of barrier precautions 